\title{
Minkowski Space and Tachyon Velocity
}

\author{
Jacques Consiglio ${ }^{1}$ \\ ${ }^{1}$ Labastidette, France \\ Correspondence: Jacques Consiglio, 52, Chemin de Labarthe, F31600 Labastidette, France. Tel: \\ +33-687-493-664. E-mail: jacques.consiglio@gmail.com
}

Received: July 5, 2013 Accepted: July 25, $2013 \quad$ Online Published: October 9, 2013

doi:10.5539/apr.v5n6p1

URL: http://dx.doi.org/10.5539/apr.v5n6p1

\begin{abstract}
A brief analysis of classical tachyons in Minkowski space shows that de Broglie wave phase velocity $V=c^{2} / v$ does not enable causality violations and gives an inversion of energy/momentum ratio between bradyons and tachyons. It leads to considering $V v=c^{2}$ as a symmetry of Minkowski space where, for $V>c$, the signature of space-time is seen inverted. The inversion is shown coherent with Cramer's transactional interpretation of quantum mechanics, but implies the physical existence of the past and the inexistence of the future.

In a recent paper we assume that the electron wave is a magnetic current at the origin of its mass; it leads to reversed coefficients with respect to the Dirac condition. Using space-time signature inversion we show that the monopole has, in linked electrons states, quantized velocities in agreement with the de Broglie wave phase velocity. The magnetic charge is relativistic and quantum in origin and comes from the Lorentz transformation of a quantum of current. We also show that gravitation is a natural consequence of the theory.

The predicted charges agree with experimental results from Ehrenhaft, Schedling, Ferber and Mikhaïlov. They also agree, in phenomenology, with recent results related to symmetry breaking in superconductors, and give plausible kinematics to the emission process in the Podkletlov and Poher experiments.
\end{abstract}

Keywords: Minkowski space, de Broglie wave, tachyons, magnetic monopole, origin of mass, interpretation of quantum mechanics, Podkletnov IGG, Poher Universons, gravitation

\section{Introduction}

In a recent paper (Consiglio, 2013) we propose a new quantization of magnetic poles, resulting in reversed coefficients with respect to the Dirac condition. This result appears incoherent with quantum physics and then the initial object of this study is to continue the analysis in the direction of classical tachyon monopoles. But our analysis of classical tachyons goes far beyond. It shows that de Broglie wave phase velocity has quite specific and interesting properties:

- Although it can appear contradictory, it does not enable causality violation by tachyons exchange.

- It gives an inversion of the Energy/Momentum ratio between bradyon and tachyon.

- It implements the Feynman-Stuckelberg reinterpretation principle and the related switching procedure.

- It can be interpreted as symmetry of Minkowski space where, for $V>c$ the signature is inverted.

This symmetry gives a complimentary de Broglie wave in one space dimension (the usual time) with three time dimensions (the usual space), which naturally follows the particle trajectory in the electromagnetic field. The resulting concept is shown in agreement with Cramer's transactional interpretation of quantum mechanics.

We then address our initial subject of tachyon monopoles. In agreement with our initial quantization (Consiglio, 2013), we show thru different approaches that the monopole velocity is the de Broglie wave phase velocity, and we find that the monopole is a quantum of current: $g V=e c / 2$. The quantum is also deduced from and agrees with the inversion of space-time signature in the tachyon domain. It makes the monopole simultaneously quantum and relativistic in origin. It results in symmetric Maxwell equations where the magnetic current is a quantum while the magnetic charge is a relativistic effect, the opposite of the electric charges and currents. The magnetic current is then invariant and, contrary to classical tachyon theories, a Lorentz boost cannot give an infinite current. We notice that since Dirac's quantization is entirely based on the Copenhagen interpretation of quantum mechanics, the existence of the magnetic charge that we predict probably invalidates this interpretation. 
Finally, the theory leads to gravitation as a necessary effect of the inexistence of the future, while the past must have a physical existence: it must be built "now" and the building process creates some depletion in the mass-giving field. It leads to the emergence of a pseudo-metric in flat space.

We apologize for not referencing all the literature on classical tachyons; systematic references would make our paper uselessly long and barely readable. Most relevant ones can be found in the two papers we quote. We do not discuss quantum tachyon theories, because at this stage it is necessary to re-understand basic quantum mechanics in light of our results - we address the Bohr model and show how it can be understood using magnetic currents.

\section{On Tachyons}

\subsection{Mass and Classical Tachyons}

In this section, we first assume that tachyons are massive superluminal particles with an imaginary mass $m_{t}$. Classical tachyons theories are based on the analysis of Einstein's $E^{2}=m^{2} \gamma^{2} c^{4}=m^{2} c^{4}+P^{2} c^{2}$, for $V>c$. The usual Lorentz transformation applies and defines three exclusive domains separated by light speed: so called bradyon, luxon and tachyon (so that for instance a tachyon is in his domain for any subluminal observer).

Energy and momentum evolve in a mirror manner in the tachyonic domain with respect to bradyons. Precisely, tachyons momentum approaches an asymptotic non null limit $\left(m_{t} c\right.$, with $m_{t}$ imaginary) when their velocity increase, while their energy approaches zero. Both increase indefinitely when their velocity approaches (slows down to) light speed. Then based solely on special relativity, this makes tachyons fields perfect candidates to the origin of particles mass, because we can define a symmetry or a correspondence between tachyons energy and bradyons momentum on one hand, and between tachyons momentum and bradyons energy on the other hand.

Denoting $\gamma_{S}$ and $V$ for superluminal velocities, with $\gamma_{S}$ defined in positive range $\left(\gamma_{S}^{2}=1 /\left(V^{2} / c^{2}-1\right)\right)$, and $\gamma$ and $v$ for subluminal particles, we can always define a subluminal velocity $v$ so that $v V=c^{2}$; it authorizes to express the tachyon energy $E$ and momentum $P$ in two symmetrical manners given by:

$$
\begin{gathered}
E^{2} / c^{2}=m_{t}^{2} \gamma_{S}^{2} c^{2}=m_{t}^{2} \gamma^{2} v^{2} \\
P^{2}=m_{t}^{2} \gamma_{S}^{2} V^{2}=m_{t}^{2} \gamma^{2} c^{2}
\end{gathered}
$$

where $m_{\mathrm{t}}$ is the tachyon proper mass. The symmetry above in $E^{2}$ and $P^{2}(1.1-1.2)$ shows a natural correspondence between subluminal and superluminal velocities, which justifies the following conjecture:

a) Tachyon momentum is bradyon energy.

b) Tachyon energy is bradyon momentum.

On this basis, let us denote $m_{b}$ an element of a bradyon mass, which is "given by" the momentum of a tachyon of mass $m_{t}$ (or conversely). Our conjecture leads us to assume a general law of transformation of the tachyon spectrum that will make the symmetry invariant giving:

$$
m_{b} \gamma c \quad \leftrightarrow \quad m_{t} \gamma_{S} V
$$

But (2) can be valid as a general law solely if $\gamma c=\gamma_{S} V$ for any observer, which implies $V v=c^{2}$. It shows that, in the frame of our conjecture, a tachyon is linked to its source. But if a law of transformation exists and agrees with (2), it cannot be based on mass or energy, it needs some underlying quantity that we will denote $k$; then, considering that in (2) the $\gamma$ and $\gamma_{S}$ are related to time dilation, if a tachyon "is" an element $m_{b}$ of a bradyon proper mass-energy, since $m c$ is invariant we should have, at least in the non relativistic case:

$$
k V=\text { const. }
$$

Then from (1.1-1.2), the transformations of energy and momentum come naturally as a consequence.

\subsection{Causality in Special Relativity}

Modanese (2013) analyze the conditions for passive causality violation and momentum inversion. He proves that they are exclusive of each other because they occupy two domains on each side of a curve defined by $v / c=c / V$. But how could momentum revert without an inversion of the velocity? How could this fit with the conservation laws? The logical answer is that tachyons are "on the curve" $v V=c^{2}$, and then the tachyon direction and momentum reverses like the direction of its source. Modanese also shows that "if a source S emits a tachyon which is absorbed by a non-relativistic target particle T with energy-momentum ratio $E / P=\left(m v^{2} / 2\right) /(m v)=v / 2$, the $E / P$ ratio of the tachyon must be equal to $v / 2$ and therefore the target determines the tachyon propagation velocity". It agrees with our conjecture and then we must use the bradyon $E / P$ ratio in a relativistic form; it gives:

$$
E / P c=m \gamma c / m \gamma v=c / v
$$


Then from (4), if the tachyon "is linked to its source by $v V=c^{2}$ ", the relativistic E/P agreement between the source and the target is universal. Each sees the other emitting tachyons with the same E/P ratio, but we cannot consider those transfers as simple particles exchanges; there must be something below.

From (1.1-1.2), the tachyon energy-momentum ratio naturally verifies: $E / P c=c / V=v / c$. It is the opposite of (4) in agreement with our conjecture. It shows that the de Broglie wave phase velocity, $V=c^{2} / v$, is natural in special relativity when considering tachyonic exchanges between bradyons.

Importantly, this velocity puts an end to causal paradoxes. The reason is that we always have $\boldsymbol{V} \cdot \boldsymbol{v}>0$, with $\boldsymbol{V}$ and $\boldsymbol{v}$ parallel vectors; it means that for a given target, $\boldsymbol{V}$ is directed like the velocity of the source, and then for any observer it propagates toward to its future. No material system can ever send a signal backward in time, because according to special relativity no observer can see de Broglie waves propagate toward the past. Interestingly, this result is somewhat already in the literature, but in a different form: According to Recami (1985), all causal paradoxes can be solved using the Feynman-Stuckelberg reinterpretation principle, and the associated switching procedure (see Recami, 1985, for an exhaustive review). The procedure goes as follows: If a given observer sees a tachyon going backward in time, we must switch it to an anti-tachyon going forward in time. It can pose conceptual problems if we assume that tachyons velocity can be anything (like in all causal paradoxes so far). But the procedure is naturally done by $V v=c^{2}$, and then we have no need for a principle.

\subsection{Minkowski Space}

Let us now interpret this result. In other words, what does it imply concerning Minkowski space?

It has been known since de Broglie (1924) that $V=c^{2} / v$ is compatible with the Lorentz transformation. Consider two particles or systems $\mathrm{E}$ and $\mathrm{R}$, with relative velocity $v$; $\mathrm{E}$ emits tachyons which interfere with $\mathrm{R}$. For an observer following R, the tachyon velocity is $V=c^{2} / v$.

For a different observer of velocity $w$ with respect to $\mathrm{R}$, the velocity of $\mathrm{E}$ is $v^{\prime}=c^{2}(v+w) /\left(v w+c^{2}\right)$; then the tachyon velocity is $V^{\prime}=c^{2} / v^{\prime}=c^{2}(V+w) /\left(V w+c^{2}\right)$. This is the Lorentz transformation of $V$ and the tachyon and bradyon velocities transform together; it shows, if needed, that $V=c^{2} / v$ is natural for bradyons tachyons interaction in special relativity, but tachyons and bradyons cannot be considered independent particles. It is also important to notice that no other general velocity formula linking tachyons-bradyons exchanges will give a more natural group of transformations (if any other does exist). We also have $v / V=v^{2} / c^{2}=\beta^{2}$, and $1 / \gamma^{2}=1-v / V$, so we can write for bradyons:

$$
\begin{aligned}
& P^{2}(1-v / V)=m^{2} v^{2} \\
& E^{2}(1-v / V)=m^{2} c^{4}
\end{aligned}
$$

But then, what is the physical significance of $V=c^{2} / v$ ?

Consider a source A "emitting" tachyons and co-located receivers B and C, of respective velocities $v_{B}$ and $v_{C}$ in the direction of A. If $v_{B} \neq v_{C}$, the Lorentz transformation shows that any observer will see the same source and targets. It seems natural but it is a true problem, because in the non-relativistic case $V>>c$, the time of travel is:

$$
T=L / V=L v / c^{2} \rightarrow L / c=c T / v
$$

Therefore, with respect to $v$ (and then for any bradyon), in (6) the quantity $L / c$ represents a dimension of time and $c T$ a dimension of space. Using $\tau=c t$, the usual Minkowski space-time coordinates can be defined as:

$$
(i \tau ; r)
$$

Then for tachyons the signature must be inverted, and two inversions are possible:

$$
(i \tau ; r) \rightarrow(i r ; \tau) \text { and }(-i r ; \tau)
$$

Consequently $V v=c^{2}$ gives a symmetry of Minkowski space.

\subsection{Symmetries of the De Broglie Wave}

Based on the analysis in the previous sections, we now assume that tachyons are not independent particles, but a manifestation of usual particles agreeing with the de Broglie wave phase velocity (i.e. we do not need a-priori to assume a standing wave). We do not know their nature but we can study the symmetry of the wave.

An electric charge reverses by $T$ symmetry. Then from (7), since $i r$ represents a dimension of time, its charge seen in the tachyon domain will reverse by parity $(i r \rightarrow-i r)$.

Let us denote $g$ this charge, we have:

$$
P: g \rightarrow-g
$$


Then a charge $g$ is not the antiparticle of a charge $-g$. Using the CPT theorem, CPT: $g \rightarrow g$, and then we have CT: $g \rightarrow-g$. As shown by Curie (1894), this is the symmetry of magnetism (incomplete because we do not have the $\mathrm{C}$ and $\mathrm{T}$ transformations).

If an observer sees an electron with velocity $v$, we can reverse the picture with a Lorentz boost that gives $-v$, and then recover the initial picture looking at the boost from the back. This is also a property of the magnetic field of the electron. If the electron wave is related to its magnetic field, the charge $g$ is proportional to $e v / c$, and then using $v V=c^{2}$, we can write $g=k e v / c \rightarrow g c / v=g V / c=k e=c o n s t a n t$, in agreement with (3).

Let us now look at the symmetry in its dimensional aspect. In classical physics, the concept of space is seen as a fixed area with three dimensions while time is a dimension of change/evolution. Then we are used to imagine a given particle at location $\mathrm{A}$ and time $\mathrm{T}$ in a given state, and in ( $\left.\mathrm{A}^{\prime}, \mathrm{T}^{\prime}\right)$ possibly in another state (for instance a different momentum or energy), and we can have $A=A^{\prime}$ with $T \neq T^{\prime}$. A particle can be at the same space coordinates at two different times, and the opposite is impossible. But now such situation is possible and even mandatory using equation (7.2), since $i r$ and $-i r$ correspond to three dimensions of time and $\tau$ is one dimension of space. This gives a symmetry that can be described as follows: A particle or a material object manifests in the form of bradyon and tachyon in different "domains": The usual Minkowski space of signature $(r ; i \tau)$ in $(7.1)$, and two versions of Minkowski space where the signature is inverted to $(i r ; \tau)$ and $(-i r ; \tau)$ in $(7.2)$. We will, for convenience, denote B-space (bradyon) and T-space (tachyon) the first and second domains, even though the separation is artificial.

The symmetry leads to an important consequence: De Broglie wavelength is experimentally verified, its frequency is $v=E / h$, its wavelength is $\lambda_{D}=h / p$, and in theory its phase velocity is $V=c^{2} / v$. It is the tachyon component of all particles. In T-space, using (6) its wavelength becomes Compton's $\lambda_{C}=h / m c$, the phase velocity becomes a group velocity $v$, and the particle pulsation (its Compton wavelength in time) is then seen as a de Broglie wave. The particle trajectory is seen as waves from the two inverted domains, it is seen as matter waves of wavelength $\lambda_{D}=h / p$, and phase velocity $V=c^{2} / v$, along the particle timeline. The wave, as seen from T-space has the same properties as in B-space, but space and time are inverted. Since the two inverted domains have one single dimension of space $(\tau)$, it implies that a particle trajectory is hosting some signal going forward and backward in time; because of the two inversions it must be both ways. Actually, in a de Broglie-like approach, the signal is a phase transported by the particle pulsation along its trajectory, which is seen from the inverted domains as two de Broglie waves thru time, one in each direction.

Consequently, the assumption that solely de Broglie wave phase velocity exists in the tachyon domain implies the absence of causality violation, but also that a particle "trajectory" hosts information transmission in the two directions of time; schematically, the particle "now" is the terminal and its trajectory is the communication line.

\subsection{Quantum Causality}

It is trivial that the conclusion of the previous section implies some (retro) action of the past and future on the state of particles at any given time (it is even quite doubtful that state and time are the right word). This result is coherent with Cramer's (1986) transactional interpretation of quantum mechanics. We recall that it is considered not testable, as it is "solely" an interpretation of the formalism; it does not predict any new experimental result, and then we could end the discussion on this point. We shall see however in the next section that the inversion of space-time might be testable (and in our opinion it might already be verified), but for this we first need to model the electron trajectory as waves and currents.

From one of the inverted space-times, an electron is seen as a current along one dimension of space. This current is $-e c$, and from the other inversion, it is seen as a current $+e c$. Since those currents can be seen as de Broglie waves and physically identical, they should be written in the form of a two components standing wave:

$$
\psi(\tau)=(-e c / 2)\left(\psi^{+}(\tau)+\psi^{-}(\tau)\right)
$$

Where $\psi^{+}$and $\psi^{-}$are complex functions, and $(e / 2) \psi^{+}$and $(e / 2) \psi^{-}$represent real currents of electricity (in usual space-time) going forward and backward in time respectively. The coefficient $e / 2$ is mandatory since we must have $\psi^{+}(\tau)>0$ and $\psi^{-}(\tau)>0$ (each current is unidirectional) and the two functions variations are unitary. In the case of an electron, $\psi^{+}$is a positive current of negative charge going forward in time, and $\psi^{-}$is a positive current of positive charge going backward in time. From this description, and using the symmetry of electricity, we see that if the current $\psi^{+}$follows a given trajectory in the presence of an electric field, the current $\psi^{-}$will trace back the same trajectory; the symmetry of magnetism $(\mathrm{T}: \mathrm{B} \rightarrow \mathrm{B}$ ) also gives the same behavior in a magnetic field.

Thus we find a possible difference with Cramer's interpretation: If two particles are close enough at a given point in space-time, their respective currents $\psi$ can influence each other locally (and information melts), which 
naturally leads us to consider entanglement. In any entanglement experiment the particles are co-located at least once during the experiment, and a local communication is possible at this place. Essentially, Cramer's offer and response waves are the $\psi^{+}$and $\psi^{-}$, and we can say that the particles' "second wave" is permanently doing the transactional job; provided the signature inversion, such communication is possible and intuitively natural.

Although this logic needs further study and verifications (consider for instance the 4 components wave in Dirac's equation with respect to $\psi^{+}$and $\psi^{-}$), we can already state that the second wave transforms according to Lorentz (as it follows the particle trajectory), and that it does not suffer any problem of dispersion and signal to noise ratio which are crucial if we want to explain entanglement using solely faster than light communication; the full "power" of $\psi^{+}$and $\psi^{-}$is permanently localized exactly like the particle: the theory is not truly non-local, it is solely symmetrical in time. It is also intuitively obvious that the concept can explain all entanglement experiments. For instance, delaying and randomizing the choice in a quantum eraser experiment will have no effect, because the state of the experimental setup must be taken into account in four dimensions.

The symmetry also gives interesting insights to the foundations of quantum processes. Firstly, the non-commutation of momentum and position operators gives $[p, x]=i$ in $\mathrm{B}$-space but then also in T-space, and $[p, x]=i \hbar$ in $\mathrm{T}$-space reads $[E / c, \tau]=i \hbar$ in $\mathrm{B}$-space and it agrees with our conjecture on the opposite $E / P$ ratios of bradyons and tachyons. In both cases (B-space and T-space), uncertainty relates to a physical action and then to the properties of the forces at work.

Secondly, we face the interesting possibility that the past can be changed (even though it is not part of Cramer's interpretation); but then how much can it change? Consider two entangled electrons and assume that the state of each is undetermined but eventually coherent. When one electron is measured it needs, in the worst case, to send a signal backward in time that will change the phase of the second electron currents (its $\psi^{+}$and $\psi^{-}$) at worst in $\pm \pi$; it requires no energy/momentum transfer but solely an action $\leq h / 2$. So the answer is trivial. If past events can be changed, it is at worst within quantum uncertainty, and it also applies to all conjugate variables. In a classical view, uncertainty now looks like a minimal conspiracy of nature, a sort of provision for the consistency of successive events; but the theory requires that the past physically exists, but not (yet) the future. This is intuitively acceptable and even natural.

\section{Tachyon Monopole}

\subsection{A Classical Approach}

The original motivation for the search of magnetic poles is the dissymmetry of Maxwell equations. Recami and Mignani (1976, Equation 12 below) state that superluminal electric charges give the missing symmetry if we confine observations to subluminal frames; using ' $S$ ' for superluminal electric charges and ' $s$ ' for subluminal:

$$
\begin{gathered}
\boldsymbol{\nabla} \cdot \boldsymbol{D}=\rho(s) \\
\boldsymbol{\nabla} \cdot \boldsymbol{B}=\rho(S) \\
\boldsymbol{\nabla}^{\wedge} \boldsymbol{E}=\boldsymbol{j}(S)-\delta \boldsymbol{B} / \delta t \\
\boldsymbol{\nabla}^{\wedge} \boldsymbol{H}=\boldsymbol{j}(s)+\delta \boldsymbol{D} / \delta t
\end{gathered}
$$

This set of equations naturally agrees with the inversion of space-time discussed in the previous section as it shows that for $V>c, \boldsymbol{H}$ and $\boldsymbol{E}$ have symmetrical roles as well as $\boldsymbol{D}$ and $\boldsymbol{B}$. As usual, $\boldsymbol{H}$ in (10.4) results from the Lorentz transformation of scalar electric charges viewed as the electric current density $\boldsymbol{j}(s)$.

At the opposite, $\boldsymbol{E}$ in (10.3) results from $\boldsymbol{j}(S)$ which from (10.2) must be a current density of pseudo-scalar charges, as required by the symmetry of magnetism. Moreover, assuming the charge $S$ is a constant of nature, there exist subluminal observers for which $\boldsymbol{j}(S)$ is infinite, and this is hardly acceptable. Even if " $-\delta \boldsymbol{B} / \delta t$ " cancels the infinite a current density must be finite for any observers. Thus the superluminal charges $S$ should transform; it shows that tachyon monopoles are candidates to verify equation (3) and comply with the underlying symmetry.

(Since $S$ represents superluminal electric charges, the term monopole is not truly appropriate, as well as the term tachyon. We will however stick to this terminology to avoid confusion with subluminal particles.)

\subsection{The Monopole in Relativity}

In his celebrated paper on magnetic charge quantization, Dirac (1931) defines the field $\boldsymbol{H}$ of a monopole with a vector potential $\boldsymbol{A}$ such that: $\operatorname{curl} \boldsymbol{A}=g \boldsymbol{r} / r^{3}$. Using a wave function $\psi$ of the electron in the absence of external field, interpreted as a density of probability of presence, he introduces a function $\exp (i \beta)$ which is the difference between a normal wave function $\psi$ in the absence of external fields and the wave function in the presence of external fields: $\psi \rightarrow \psi \exp (i \beta)$. 
He shows that the function $\beta$ has quite specific properties; it needs no definite value at each point, it is not integrable but it has definite derivatives: $k_{i}=\delta \beta / \delta x_{i}$ with $x_{i}$ in $\{x, y, z, t\}$, which are shown independent of the chosen wave function $\psi$ and then interpreted as the effect of the "fields of forces" on the electron wave function; since for the normal wave function $\psi$, the norm $|\psi|$ represents the probability of presence of the electron, the phase variation $\beta$ gives the effects of the fields of force.

Then according to Dirac (1931, Equ. 7):

$$
\operatorname{curl}(\boldsymbol{k})=(e / h c) \boldsymbol{H} ; \quad \operatorname{grad}\left(k_{4}\right)-\partial \boldsymbol{\kappa} / \partial t=(e / h) \boldsymbol{E}
$$

Now using Stokes' theorem, the change in phase of the electron wave around a closed curve verifies:

$$
\int(k, d l)=\int(\operatorname{curl}(k), d \boldsymbol{S})
$$

where $d \boldsymbol{l}$ is a line element of the curve and $d \boldsymbol{S}$ is an element of a surface bounded by the curve. If a monopole is within a closing surface bounded by the loop, the first integral vanishes when we shrink the loop, but not the second and it makes the equality impossible. But Dirac assumes a singular line terminated by the monopole, and the continuity of the electron phase around the singular line leads to (Dirac, 1931, Equations 8, 9):

$$
2 \pi n=(e / h c) \int(\boldsymbol{H}, d \boldsymbol{S})=4 \pi e g / \hbar c
$$

This is the famous Dirac quantization condition, it gives $g=n e / 2 \alpha$. The demonstration leads to a charge which is quantum mechanical in origin and it does not address the possibility that the monopole is relativistic in origin, like the magnetic field. What then? The simplest approach is to go back to de Broglie's thesis, in which the wave is a relativistic effect. We find the following equation (de Broglie, 1924, Section VI) related to the propagation of the electron wave in the electromagnetic field:

$$
V=\left(m \gamma c^{2}+e E\right) /\left(m \gamma v+e A_{l}\right)
$$

where $A_{l}$ is the projection of the vector potential in the direction of $V$. Substituting $v=c^{2} / V$, it gives:

$$
V=V\left(m \gamma+e E / c^{2}\right) /\left(m \gamma+e A_{l} V / c^{2}\right) \rightarrow A_{l} V=E
$$

Thus the wave is carrying a total charge $\hat{g}$ such that, by symmetry:

$$
A_{l} V=E \rightarrow \hat{g} V=e c
$$

In the Bohr hydrogen model we have $e^{2} V / c=n h \mathrm{c}$; then in such linked state, from (12) we get:

$$
\text { e } \hat{g} V^{2} / c^{2}=n h c
$$

But then from (13) we can go back to Dirac's demonstration and use the classical electromagnetic field. The Lorentz force coefficient on a tachyon monopole is $F=e g\left|\left(\boldsymbol{V}^{\wedge}\left(\boldsymbol{V}^{\wedge} \boldsymbol{u}\right)\right)\right| / c^{2}$; a relativistic origin disagrees with the $k_{i}$ being independent of the wave function. Then repeating Dirac's demonstration with a velocity dependent force coefficient results in adding a coefficient $1 / 2$ in (13), and the string is given by $\boldsymbol{V}^{\wedge} \boldsymbol{u}=0$.

So basically we are simply saying that the electron wave sees the proton as a tachyon monopole, and conversely. There is not much more to say and the remainder of this section provides with different views of the same idea.

\subsection{Small Monopoles}

Let us first briefly re-explain the quantization logic. (Please refer to Consiglio, 2013 for a detailed discussion.) Here are the premises of the theory:

- (A) The electron wave is assumed a magnetic current $\beta$ and the monopole wave an electric current $\xi$. The current $\beta$ is seen as the origin of the electron mass. We first assume the following ratios are equal, and related to the interaction of charges with the field:

$$
\xi / g=\beta / e \rightarrow g \beta=e \xi
$$

- (B) The change in phase of the monopole wave during its interaction with the electron is $\pi$ and relates to the electron spin (or the anti-symmetry of the electron wave); it depends on $e$ and $\xi$.

- (C) The fine structure constant $\alpha$ addresses the interaction of charges and currents of different natures while the interaction of charges and currents of identical nature is trivial.

Then reasoning is straightforward: On the $n^{\text {th }}$ orbit of the Bohr model, the change in phase of de Broglie wave around the nucleus gives $2 \pi n$, and from (C) depends on $\alpha e \beta$, while the change in phase of the monopole in its interaction with the electron is related to the electron spin and from (B) gives $\pi$; from (C) it depends on $e \xi$, and then comparing phases, (and assuming like Dirac that they are in proportion to the fields of forces,) we find:

$$
\alpha e \beta=2 n e \xi
$$


Combining (14-15), we get:

$$
g_{n}=\alpha e / 2 n
$$

The interest of the approach is that it is not velocity dependent; even if hidden in $\beta$ and $\xi$, the velocity cancels. Then from (13-16), (and because of the $V^{2}$ in (13) we must assume the same $n$,) we get:

$$
V_{n}=n c / \alpha ; \hat{g}=2 g_{n}
$$

This is de Broglie wave phase velocity in the Bohr model; we deduced this velocity from independent physical arguments and then starting the demonstration with (13-17) is also valid and it gives (14-15-16). Essentially we consider de Broglie wave as a physical object, and then the whole question is to understand how it sees the proton. From the tachyon domain it sees the field of forces of an even Dirac monopole. Using (16-17) we get:

$$
g V / c=e / 2
$$

And $e / 2$ is also the constant in (9). Equation (18) is coherent with (3), and since the electric charge $e$ is invariant it gives the monopole charge transformation, and equation (16) is valid solely for the electron linked states in hydrogen atoms. In the general case, the wave of an electron of velocity $v$ will be seen as tachyon monopoles of charge and velocity given by:

$$
g= \pm e v / 2 c= \pm e c / 2 V ; V=c^{2} / v
$$

We find an important difference with the initial concept: We get a quantum of current $g V=e / 2$, and from (19) the monopole spectrum is continuous and charge quantization happens in the form of velocity.

\subsection{Using Symmetry and Minkowski Space Inversion}

We can also use equations (10.x) and reason directly on space-time inversion. In (10.1), $\rho(s)$ is constant for any subluminal observer, and the same should naturally apply to $\rho(S)$ in (10.2). But if we invert the signature of space-time, charge densities will be seen as current densities and conversely, simply because time is seen as space and space as time. Hence $\rho(S)$ will be constant in T-space and not in B-space; the ratio $c^{2} / v$ between the Compton wavelength (in T-space) and the de Broglie wavelength (in B-space) gives the correspondence between time intervals in the former and space intervals in the latter. Time intervals in T-space are seen as space intervals in B-space, therefore assuming a single charge $S=g$, we have:

$$
\rho(S)=g c^{2} / v=\text { const. } \rightarrow g V=\text { const. } .
$$

Thus $g V$ is a quantum of current, and using (10.3) we get $|j(S)|=$ const, but we cannot get the quantum of current reasoning solely on the symmetry (as it gives $g V=e c$ ). We need parity (7.2) and two currents to get (18). This result agrees, by definition, with the inversion of the space-time signature. It can be expressed in a trivial manner: the subluminal charge $e$ is constant and the electric current $e v$ is a relativistic effect while the superluminal current $e c / 2$ is constant and the magnetic charge $e c / 2 V$ is a relativistic effect.

\subsection{Larger Charges}

We can now check the validity of our concept and equations using the Bohr model for a nucleus of charge $Z e$. The electron velocity is $v=Z \alpha c / n$, thus using (19) we get $g=Z \alpha e / 2 n$, and it gives two charges maxima; from $v \rightarrow c^{-}$we get $Z<138$, and $\mathrm{g}<e / 2$; this is coherent with and permitted by our quantization; since the charge $g$ is quantized by the electron velocity there is no problem for any magnetic charge to interfere with any nucleus.

In our initial quantization, we imagined quantized monopoles of larger charges which correspond to a change in phase of the electron wave multiple of $2 \pi$ instead of $\pi$ for the other series. But it is not justified in the Bohr model as we get $g=Z \alpha e / 2 n$. At the opposite, if we repeat the Dirac quantization with an atom nucleus of charge $Z e$, we get $g=n e / 2 Z \alpha$ (replacing $e$ by $Z e$ in (11)). The minimal charge is $g>e / 2$ for $Z<138$ and we find the same limit as before, but logically one is a minimum while the other is a maximum. But in reality, we see that the minimal Dirac charge is $e / 2 \alpha$ because the lesser charges could not interact with an electron - still they can interact with a nucleus of charge $Z e$; a circumstantial charge looks unphysical.

Lochak (2004) also remarks that the Dirac condition might not be so general as usually thought, but he concludes that a monopole is not a particle like the others and might take different charges when interacting with different nucleuses. But actually his theory gives a massless monopole (Lochak, 1997-2005), its velocity is $c$ and then the theory also gives a quantum of current. We believe that our logic fixes the problem, because we have:

$$
E=h v=4 \pi e g_{D} v / c
$$

which addresses a quantized magnetic current $g_{D} v$. The $4 \pi$ gives quantization in action $h$, and $g_{D}$ gives the 
quantization of angular momentum $\left(e g_{D} / c=h / 2\right)$. But it relates to a current, not to a charge.

Equation (21) leads to a second approach. Magnetic poles are usually assumed massive and we recall that their mass $m_{g}$ should be estimated from the charge self-interaction (or from its interaction with the field) using the electron mass $m_{e}$ (Barut, 1979; Salam and Tiomno, 1959; Stephenson, 1957):

$$
m_{g}=m_{e} g^{2} / e^{2}
$$

But we must consider the tachyon made of two currents in the same manner as an electron charge is made of two currents in (9), and then we must use $2 g$ in (22). When interacting with an electron, the conservation of energy implies that the monopole gives to the electron its equivalent self-energy. From (21-22) we get:

$$
4 \pi e g_{D} v / c=4 h v g^{2} / e^{2} \rightarrow g=\alpha e / 2
$$

Since the electron frequency $v$ cancels, this reasoning gives the same result using any reference particle having an elementary charge (a muon or a proton for instance). Moreover (22) is also valid in the Bohr model for any nucleus charge, and the energy loss corresponds to two monopoles. We have $g=Z e / 2 n \alpha$, and from (22) we get:

$$
\Delta E=2 m_{e} c^{2}(Z \alpha e / 2 n)^{2} / e^{2}=\left(Z^{2} E_{0} / 2 n^{2}\right)
$$

... as expected. For a muon, replace $m_{e}$ by the muon mass.

\section{Gravitation}

The two currents $\psi^{+}$and $\psi^{-}$of (9) come from the past and the future. But logically the future does not exist and then at least one of them must be built "on the fly" and frozen in space-time as an emission of charges $e / 2$. Therefore any massive particle is a pit absorbing a total charge $g_{A}(e / 2)$ at each period of its pulsation, which must physically come from an existing field of undetermined charges $g_{U}$ constituting a constant background current $\Phi_{0}$. It will create the equivalent of an isotropic negative current $g_{A} v / 4 \pi R^{2}$ in its own direction. The inertial and gravitational masses of any massive body are proportional to its frequency $v=m c^{2} / h$. Hence in a void space with a static central mass $M$ of frequency $v_{M}$, the resulting current $\Phi$ is given by:

$$
\Phi=\Phi_{0}-g_{A} v_{M} S / R^{2}
$$

where $S$ is a constant of nature (a cross-section in $\mathrm{m}^{2} / \mathrm{Hz}$ ). So we see that the field cannot work trivially in energy because the $1 / R^{2}$ in (25) corresponds to an attractive force. But it agrees with our conjecture that tachyons momentum is bradyons energy, and then equation (25) is equivalent to a pressure field in $1 / R^{2}$ that gives the Newton potential; except for some numerical coefficients we get:

$$
\Phi-\Phi_{0} \rightarrow \Gamma=-g_{A} v_{M} S / R+\text { const. }
$$

But obviously the current density at the test particle location will determine its proper frequency, as it is the rate of absorption of the charges $g_{A}$, and then also its Compton wavelength; hence we can use conventional equations. A test particle energy and pulsation will be:

$$
E=E_{0}\left(1-M G / c^{2} R\right) \rightarrow v=v_{0}\left(1-M G / c^{2} R\right)
$$

Our analysis implies that all energies will be impacted by (27), not only a test particle but also any measurement instrument. Then clocks and rulers at different distances from the central body will be seen differently by any observer. From (27) we get:

$$
d L_{l}^{2}=d L_{0}^{2}\left(v_{0} / v_{l}\right)^{2} ; d T_{1}^{2}=d T_{0}^{2}\left(v_{1} / v_{0}\right)^{2}
$$

In the weak field approximation $\left(1>>M G / R c^{2}\right)$, using (27-28) we get:

$$
d S^{2}=c^{2} d T_{1}^{2}-d L_{1}^{2}=c^{2} d T_{0}^{2}\left(1-2 G M / R c^{2}\right)-d L_{0}^{2} /\left(1-2 G M / R c^{2}\right)
$$

Equation (29) is the Schwarzschild metric. It shows that a pressure field "creating the past" is coherent with all verified results of general relativity, except maybe for some cosmological aspects. This result needs a few comments as it also addresses different theories, and not only general relativity:

- The field is a positive pressure field similar in effect to Poher's Universons which was shown coherent with general relativity (Poher \& Marquet, 2012).

- In a precedent paper (Consiglio, 2012) we analyze Poher's hypothesis and we get the equivalent of the Schwarzschild metric as an emergence (or an appearance) in flat space; it is not a metric theory.

- Marquet (2013) shows that a pressure field, together with a non-Riemannian curvature removes the main pathological aspect of general relativity: the Einstein pseudo-tensor becomes a true tensor.

The coherence of the approaches is obvious, and equation (29) shows that a magnetic current is pertinent. 
But the field density will be affected by the universe expansion. Contrary to bradyons and luxons, an expansion will increase the tachyons energy and momentum (in our case, the monopoles charge) as their apparent velocity will get closer to the speed of light. Therefore the field velocity "now" in free space must be very close to the speed of light (and gravitation is equivalent to a shadow effect). As shown by Hafele (2012) this velocity is coherent with the flyby anomaly reported by NASA in 2008 and the anomalous decrease in the Moon's orbital speed. Obviously, it will give additional anomalous effects at the opposite of current theories, to begin with a permanent growth of the universe total energy; a self-accelerating expansion seems natural.

There are other specific effects that we will not discuss as they out pass the scope of this paper.

\section{Some Experimental Aspects}

\subsection{Magnetic Charges Measurements}

Let us first recall that direct measurements of Ehrenhaft (1930, 1944), Schedling (1950), Ferber (1950) lead to an estimate of the magnetic charge $g=\alpha e$; Mikhaïlov $(1982,1985,1987,1991)$ obtained similar results, but using modern data analysis tools and from a large number of samples he gave an empirical estimate of the magnetic charge quantum $g=\alpha e / 6$. Both estimates are consistent with our quantization.

Until now, the whole set of experiments could not be explained without the presence of magnetic charges, but no convincing explanation exists giving the nature of the charges and some of their properties; not to mention the discrepancy with the charge predicted by Dirac $\left(3 / \alpha^{2}>56000\right)$. The theory in this paper explains the measured charges and the low energy production, but also other experimental effects.

The first is systematic in all experiments and is worded simply by Mikhaillov: "The effect stops almost instantly when we the illumination (of the aerosol particles) ends". Take the example of a monopole of charge $\alpha e$, its theoretical velocity is $V \approx 68.5 \mathrm{c}$, and it should not be observed; but its interaction time with an electron is indirectly given by (21) $T \approx 10^{4} / v \approx 10^{-16} \mathrm{~s}$, since its action must be $h$.

Hence, without computing the monopole average time to escape from the aerosol particle, it is obvious that it will depend on its mass $m$ and of its radius. If, for instance, it interferes with $10^{16}$ electrons it will escape after one second. It shows that even with small aerosol particles the escape time should be measurable; it would then be of interest to have an estimate of the "almost" and of its dependence on the mass of the aerosol particle.

The second effect is that the observed charge is proportional to the light intensity. It is trivially explained in the same manner as the light intensity gives the charges production rate while the retention time should solely depend on the aerosol particle mass.

The third effect concerns an experiment of Mikhaïlov in which the ferromagnetic aerosols were in water droplets. The mass of the particle was increased in a factor $10^{6}$, and the observed charges increased around $10^{3}-10^{4}$ (though not yet up to a Dirac charge). It agrees with an escape time dependent on the the particle mass.

The fourth effect is the most stunning; Mikhailov noticed that when an aerosol particle approaches the walls of the experimental chamber it stops before touching it and find a point of equilibrium. It shows in our view that the magnetic charges permanently escape from the particle and are retained by the chamber walls (from which they slowly escape); this in turn creates a repelling force on the particle.

The sole criticism of Mikhaillov results found in the literature was made by Akers (1988), who finds that Mikhaïlov results are consistent with a Dirac charge. Let us prove that his reasoning is theoretically correct but irrelevant with respect to the experimental data.

Mikhailov repeats and improve Ehrenhaft experiments with ferromagnetic aerosols; in particular, he applies alternative electric and magnetic fields (which are orthogonal to each other) to aerosol particles with suspected dual charges. The particles trajectories and their dependence on the applied fields give the ratio $g / e \approx n \alpha / p$, with $n, p$ small integers.

Akers' reasoning is based on the fact that Mikhaïlov does not measure any movement along the axis orthogonal to the electric and magnetic fields, and then Ackers (1988, Equation 5-6) states that the following forces are always equal and of opposite direction:

$$
e \boldsymbol{v}^{\wedge} \boldsymbol{B}=g \boldsymbol{v} \wedge \boldsymbol{E} \rightarrow e / g=E / B
$$

Technically, the force expression needs $\beta$, not $v$. Since Mikhaïlov measures movements under one millimeter and uses field pulsations of a few hertz, $v$ is very small; say $v<1 \mathrm{~m} / \mathrm{s}$ and then $\beta<1 / \mathrm{c}$, and since the particle accelerations in the electric and magnetic fields are in the same range $(g B / e E \rightarrow 1)$, we can estimate a ratio of forces-for instance between $e \boldsymbol{E}$ and $g(\boldsymbol{v} / c)^{\wedge} \boldsymbol{E}$. Using $g=\alpha e$, we find $g \beta / e<\alpha / c \approx 2.410^{-11}$. 
Since $e \boldsymbol{E}$ gives movements around $1 \mathrm{~mm}$, Akers requires Mikhaïlov to measure $2.410^{-14} \mathrm{~m}$, on a free micron-size aerosol particle moving of $1 \mathrm{~mm}$, which of course is impossible. It is then obvious that (30) cannot be positively verified. Note that using a Dirac charge $g=e / 2 \alpha$ gives similar results, and then no discrimination is possible.

Then Akers re-analyze Mikhaïlov's results in light of (30): From measurements of $e E / g B$, Mikhaïlov finds typically $g / e \approx n \alpha / p,(n, p$ small integers, often equal to $l)$ and then using (30)-which cannot be experimentally tested - and the same data, $e B / g E$ will trivially give the opposite ratios and classical Dirac charges.

The whole logic developed by Ackers is then irrelevant at low energy.

\subsection{Cooper Pair mass anomaly in Niobium}

Since we assume that the electron mass is a magnetic current, the anomalous Cooper pairs mass in Niobium measured by Tate et al $(1989,1990)$ is relevant to our logic as we should find quantized monopoles giving the mass excess. As far as we know, there currently exists no QED explanation giving a perfect prediction. The standard QED computation gives a mass defect, while the experiment shows a mass excess; denoting $m$ ' the Cooper pair mass and $m_{e}$ the electron mass, we have (Tate et al. 1989, 1990):

- QED: $\left(m^{\prime} / 2 m_{e}\right)=0.999992$.

- Experiment: $\left(\mathrm{m}^{\prime} / 2 \mathrm{~m}_{e}\right)=1.000084$, with an accuracy of $21 \mathrm{ppm}$ and a precision of $5 \mathrm{ppm}$.

In our logic, the excess mass should be given by monopoles involved in the Cooper pairing process. An empirical search based on (21) and $\left(m^{\prime}-2 m_{e}\right) c^{2} \approx 2(4 \pi e \Delta g v / c)$, where $v$ is the electron rest frequency gives:

$$
\Delta g \approx 3 \alpha e
$$

It corresponds to a difference of $6.5 \mathrm{ppm}$ with respect to the measured mass, in the range of the measurement accuracy. Now using (22) and $\Delta m=2 m_{e} \Delta g^{2} / e^{2}$, we get:

$$
\Delta g \approx 1.13 \alpha e
$$

Another plausible explanation is that the Cooper pairing requires a velocity giving $\gamma=m^{\prime} / 2 m_{e}$ (in a manner assumed similar to the Bohr model, but linking two electrons together with the crystal lattice). It gives $v \approx c / 66$, which is quite close to $c / 68.5 \rightarrow V \approx 68.5 c$, the theoretical velocity of the monopole $\alpha e$.

Although the three results ( $3 \alpha e, 1.13 \alpha e$ and $\alpha e$ ) seem to disagree, the three coincidences are quite stunning.

\subsection{Podkletnov Impulse Gravity Beam}

A true breakthrough would be to measure a superluminal group velocity consistent with the predicted spectrum; but such experiment is forbidden in almost all existing theories as it would result in superluminal energy transfers. As far as we know, except for quantum causality experiments, we find only one that provides evidence. Its results were published by Podkletnov and Modanese (2012) in a recent book and are not reproduced at this time (see also Podkletnov \& Modanese, 2003). Let us briefly recall the main results:

a) The apparatus produces a focused beam of unknown nature, which provokes a strong and brief distant acceleration of matter (a push). The acceleration is estimated to $>10^{4} \mathrm{~m} / \mathrm{s}^{2}$; it is independent of the target composition (to the extent of the measurement precision) and depends on the discharge energy.

b) It does not produce any heat in the targets (to the extent of the measurement precision).

c) The beam goes thru solid matter, including walls, without noticeable change in effect.

d) A fast beam component propagating at $64( \pm 1) c$ is measured directly by impact on two piezoelectric detectors separated by $1211 \mathrm{~m}$. The propagation delay is $63( \pm 1) \mathrm{ns}$; it is constant, independent of the discharge energy and of the emitter (while the beam intensity is dependent).

e) A second component is deduced indirectly at velocity $c$ from the duration of photons scattering by the beam, with an angle of 0.1 degrees, and over a distance of $57 \mathrm{~m}$. The pulse is estimated to last $\approx 40 \mathrm{~ns}$, from the scattering duration.

The constancy of the propagation velocity leads to consider a velocity quantization at the source (or a constant of nature which is not acceptable in special relativity).

Then assuming this is a monopole beam, using (17) we find:

$$
n c / \alpha=64 c \rightarrow n \approx 1 / 2 \rightarrow g \approx \alpha e
$$

This is possibly the same monopole as in the previous section (31) (or close as we get exactly $g=1.07 \alpha e$.). Its velocity is $V=68.5 \mathrm{c}$, which is $7 \%$ away from the $64 \mathrm{c}$, out of bound considering the measurement precision but 
not so much. We can first imagine some beam attenuation thru $1.2 \mathrm{~km}$ of air which reduces the impact of the beam and then also the measured velocity. But we can also find the effect of a rest frame in the difference between $68.5 c$ and $64 c$, and it is logical to consider the inertial frame in which cosmic microwave background (CMB) is isotropic as the privileged frame. Podkletnov states that the experiment took place near Moscow (Russia), and then we can use:

- Moscow's latitude of 56 degrees.

- The CMB dipole is seen on earth with a Doppler shift of $370 \mathrm{~km} / \mathrm{s}$ in the direction of Vega.

- The celestial pole of earth is oriented approximately 14 degrees from the CMB dipole.

In the worst case, the experiment was run day and night, and then the CMB dipole was seen with an angle to the south horizon varying between 70 and 42 degrees $(56 \pm 14)$, and a median angle of 56 degrees; Lorentz transformation of the $68.5 c$ gives the measurable velocities.

For a north-south beam, we get approximately:

$$
\text { Min: } 64.4 \text { c; Med: } 65.4 \text { c; Max: } 66.4 c
$$

The correction is plausible but not conclusive, since the beam orientation and the measurement date and time are unknown (and in any case, we did not take into account a probable attenuation of the beam). For instance, a south-north beam would give measurable velocities above $72 \mathrm{c}$.

The interaction of the monopole $\alpha e$ with an electron gives a change in phase $2 \pi$ of its wave and this agrees with a beam going thru matter. It explains why we get $\alpha e$ and no other charge, first because charges with higher velocity correspond to the second series and have a change in phase $\pi$ (they should be shielded by electrons), and second because charges of the same series with $g>\alpha e$ must have a lesser velocity. Podkletnov measured the front edge of the beam, and the fastest non-shielded quantized component is $\alpha e$. The monopole is the electron wave; it will not heat the targets. Then in first rough analysis, the theory seems to agree with Podkletnov results.

\subsection{Poher Experiments}

Poher (2011) reports anomalous effects of strong electric discharges in superconducting devices with a composite structure, so called emitters. They are:

A) A strong reaction force on the emitter (up to $>5 K N$ ) during the electric discharge, in the direction opposite to the electrons flow in the emitter. The recoil mometum is proportional to the electric energy of the discharge and to the surface of the emitter.

B) A distant acceleration of matter similar to that produced by Podkletnov, but a weaker dispersive beam.

C) The charging of flat capacitors in the beam.

D) The generation of an electric current in a coil of specific geometry if put in the beam.

The experiments look like a great simplification of that of Podkletnov. The main difference in results comes from the geometry of the emitters and the lower intensity of the discharge, but also, in our view, from the fact that Poher was guided by his Universons theory that predicts all the points $(A, B, C, D)$ above. Poher measures an output momentum in the range of $200 \mathrm{~g} \mathrm{~m} / \mathrm{s}$, and he estimates the mechanical losses to $80 \%$. He uses discharges of around 200 Joules where the measured electrical losses are $>90 \%$. So we see that (even with tachyons) the measured momentum is several orders of magnitude higher than permitted by the discharge energy. It implies that the emission process is pumping an existing field that can transfer momentum.

Consider now a Cooper pair accelerated to a velocity $v=\alpha c / 2$ and then, for unknown reasons, one of its electrons emits a monopole $g=\alpha e$ in the direction of the pair velocity. Denoting $m$ the electron mass, the Cooper pair kinetic energy is $E_{C}=2 m v^{2} / 2=m \alpha^{2} c^{2} / 4$; using (21), the energy loss of one electron due to the monopole emission will be $\Delta E=m \alpha^{2} c^{2} / 2$, and then $E_{C}=\Delta E / 2$. The Cooper pair momentum is given by $P_{C}=m$ $\alpha c$, and the electron recoil is $\Delta P=-2 m \alpha c$ (it can be computed directly from the Bohr model), and then $P_{C}=$ $-\triangle P / 2$. The Cooper pair is then reflected and the energy and momentum ratios are in agreement as well as the $E / P$ ratios; this is the fundamental point of our analysis of tachyons. This coincidence is a specific of the monopole $\alpha e$.

If the initial acceleration is due to an electric field the process can repeat indefinitely and the Cooper pair is stationary; then the average current is null and there is no energy expense. This is, in theory, unlimited propulsion at no cost.

In practice the electric current is quite strong, but the kinematics predicts that the effect increases with the 
surface of the emitter: This is simply because when the current density gets lower, Cooper pairs are slower and then closer to stationary. This prediction also comes in Poher's Universons theory and it was experimentally verified.

Importantly, this mechanism does not appear to break the third law of Newton, because the opposite transfer will happen sooner or later when the monopoles are absorbed by the masses they cross. But it will create some kinetic energy in the emitter and in the absorber that, logically, should come from the same field that expands the universe.

\section{Conclusions}

The double wave concept comes from a symmetry of Minkowski space-time given by $v V=c^{2}$. It does not permit causality violations but it agrees with Cramer's transactional interpretation of quantum mechanics (and it does not require action on the past to any extent larger than quantum uncertainty).

The symmetry gives a geometrical link between relativity, the phase velocity of the de Broglie wave and quantum mechanics which, perhaps, has not been fully appreciated.

It confirms our logic to monopoles quantization and gives quantized velocities in the tachyon domain that result in a quantum of current; a surprising but logical inversion between electricity and magnetism. It makes the monopole relativistic and quantum in origin, including its apparent charge, its velocity and the Dirac string.

The predicted magnetic charges agree, when quantized, with direct measurements; they also fit with some other experimental results in energy and velocity. We saw in particular that the monopole $g=\alpha e$ seems consistent thru distinct experiments, it explains the characteristics of the Podkletnov and Poher beams and it also gives plausible kinematics to the emission process; the kinematics look quite similar to the classical Cooper pair scheme.

The principles of the theory also link gravitation with electromagnetism and quantum physics. As previously shown (Consiglio, 2012), the symmetry of magnetism discovered by Lochak (1995-2007) does not require distinct fields to get the symmetries of the three forces of nature (and then later search for a unification scale and higher symmetry groups), because they can all emerge from the symmetries of electric charges and magnetic currents. As outlined in sections 5, it is also coherent with the known fact that gravitation is not a force like the three others, and we find that it does not require any additional or specific particle exchange. This is, among other reasons, why a quantum of current is of importance.

We also showed, indirectly, that measurements of superluminal velocities should be repeated with different orientations with respect to the CMB (and perhaps also experiments on anomalous masses if they use a preferred direction). Maximal differences should appear using north-south versus south-north directions, and depend on the latitude. For the same reasons, experiments using spinning devices like the detection of an anomalous London moment by Tajmar et al. (2006) should give apparently opposite parity differences at sufficiently high latitudes of the two hemispheres. It might be the easy way to prove the existence of a rest frame agreeing with the CMB. The Graham et al. (2008) results can be interpreted as a direct confirmation but there seems to be some dispute on the reality or the interpretation of the measurement (see also Lewis, 2012).

\section{Acknowledgements}

It is a pleasure to thank Giovanni Modanese and Simon Townsend for their help in the corrections of this paper and for interesting remarks and questions.

\section{References}

Akers, D. (1988). Mikhailov's experiments on detection of magnetic charge. International Journal of Theoretical Physics, 27, 8. http://dx.doi.org/10.1007/BF00669443

Barut, A. O. (1979). Lepton mass formula. Physical Review Letters, 42, 1251. http://dx.doi.org/10.1103/PhysRevLett.42.1251

Consiglio, J. (2012). On particles mass and the Universons hypothesis. Applied Physics Research, 4(2). 144-158. http://dx.doi.org/10.5539/apr.v4n2p144 Consiglio, J. (2013). Quantized charges and currents in the electromagnetic field. Applied Physics Research, 5(1), 126-134. http://dx.doi.org/10.5539/apr.v5n1p126

Cramer, J. (1986). The transactional interpretation of quantum mechanics. Reviews of Modern Physics, 58(3), 647-687. http://dx.doi.org/10.1103/RevModPhys.58.647

Curie, P. (1894). Sur la possibilité d'existence du magnétisme libre. J. Phys. Theor. Appl., 3(1), 415-417. http://dx.doi.org/10.1051/jphystap:018940030041501 
De Broglie, L. (1924). Recherches sur la théorie des quanta. Annales de Physique - 10e sérié - Tome III Janvier-Février.

Dirac, P. A. M. (1931). Quantised singularities in the electromagnetic field. Proceedings of the Royal Society A, 133, 60-72. http://dx.doi.org/10.1103/10.1098/rspa.1931.0130

Dirac, P. A. M. (1948). The theory of magnetic poles. Physical Review, 74(7), 817-830. http://dx.doi.org/10.1103/PhysRev.74.817

Dirac, P. A. M. (1978). The monopole concept. International Journal of Theoretical Physics, 17(4), 235-247. http://dx.doi.org/10.1007/BF00672870

Ehrenhaft, F. (1930). Die longitudinale und transversale Elektro-und Magnetophorese. Phys. Z., 31, 478.

Ehrenhaft, F. (1944). New Experiments about the Magnetic Current. Physical Review, 65, 62-63.

Ferber, J. A. (1950). Acta Phys. Australica, 4, 133.w.

Graham, R. D., Hurst, R. B., Thirkettle, R. J., Rowe, C. H., \& Butler, P. H. (2008). Experiments to detect frame dragging in a lead superconductor. Physica C, 468(5), 383-387. http://dx.doi.org/10.1016/j.physc.2007.11.011

Lewis, R. A. (2012). In Modanese G. \& Robertson G. A. (Eds.), Tajmar experiments on "condensing the vacuum". In: Gravity-superconductors interactions; Theory and experiments (p. 132). Bentham.

Lochak, G. (1995). The Symmetry between Electricity and Magnetism, Advanced Electromagnetism. World Scientific, Singapore. Retrieved from http://aflb.ensmp.fr/MEMOS/GLmonopole/MONOPOLE.pdf

Lochak, G. (2004). Quelques questions à propos de la formule de Dirac pour la charge d'un monopole magnétique. Annales de la Fondation Louis de Broglie, 29(4), 695-705. Retrieved from http://aflb.ensmp.fr/AFLB-294/aflb294m168.pdf

Lochak, G. (2007). The symmetry between Electricity and Magnetism and the equation of a leptonic Monopole. Fondation Louis de Broglie. Retrieved from http://arxiv.org/abs/0801.2752

Mikhaïlov, V. F. (1982). Physical Review Letters, 1303, 331.

Mikhaïlov, V. F. (1985). Observation of magnetic monopoles in the field of a line conductor with a current. Journal of Physics A, 18(14), L903. http://dx.doi.org/10.1088/0305-4470/18/14/014

Mikhaïlov, V. F. (1987). Light microparticles and magnetic charge phenomenon. Ann. Fond. L. de Broglie, 12, 491.

Mikhaïlov, V. F. (1991). Observation of apparent magnetic charges carried by ferromagnetic particles in water droplets. Journal of Physics A, 24(1), 53-58. http://dx.doi.org/10.1088/0305-4470/24/1/016

Mikhaïlov, V. F. (1993). Courants, Amers, Ecueils en Microphysique, Paris: Fond. L. de Broglie.

Modanese, G. (2013). Velocity requirements for causality violation. Retrieved from http://arxiv.org/abs/1304.5522

Podkletnov, E., \& Modanese, G. (2003). Investigation of high voltage discharges in low pressure gases through large ceramic superconducting electrodes. Journal of Low Temperature Physics, 132(3-4), 239-259. http://dx.doi.org/10.1023/A:1024413718251

Podkletnov, E., \& Modanese, G. (2012). In Modanese G. \& Robertson G. A. (Eds.), Study of light interaction with gravity impulses and measurements of the speed of gravity impulse. In: Gravity-superconductors interactions; Theory and experiments (p. 169). Bentham.

Poher, C., \& Poher, D. (2011). Physical Phenomena Observed during Strong Electric Discharges into Layered Y123 Superconducting Devices at 77 K. Applied Physics Research, 3(2), 51-66. http://dx.doi.org/10.5539/apr.v3n2p51

Poher, C., \& Marquet, P. (2012). General relativity and universons. Applied Physics Research, 4(1), 120-127. http://dx.doi.org/10.5539/apr.v4n1p120

Recami, E., \& Migneni, R. (1976). Magnetic monopoles and tachyons in special relativity. Physics Letters B, 62(1), 41-43. http://dx.doi.org/10.1016/0370-2693(76)90042-3

Recami, E. (1987). Tachyon kinematics and causality: A systematic thorough analysis of the tachyon causal paradoxes. Foundations of Physics, 17(3), 239-296. http://dx.doi.org/10.1007/BF00733092 
Salam, A., \& Tiomno, J. (1959). On the masses of elementary particles. Nuclear Physics, 9(4), 585-587. http://dx.doi.org/10.1016/0029-5582(58)90341-9

Schedling, J. A. (1950). Acta Phys. Australica, 4, 98.

Stephenson, G. (1957). A classical calculation of the nucleon-meson coupling costant. II Nuovo Cimento, 5(4), 1009-1010. http://dx.doi.org/10.1007/BF02903225

Tajmar, M., Plecescu, F., Marhold, K., \& de Matos, C. J. (2006). Experimental detection of the gravitomagnetic london moment. ArXiv, gr-qc/060303. Retrieved from http://arxiv.org/abs/gr-qc/0603033

Tate, J., Cabrera, B., Felch, S. B., \& Anderson, J. T. (1989). Precise determination of the cooper-pair mass. Physical Review Letters, 62(8), 845-848. http://dx.doi.org/10.1103/PhysRevLett.62.845

Tate, J., Cabrera, B., Felch, S. B., \& Anderson, J. T. (1990). Determination of the cooper-pair mass in niobium. Physical Review B, 42(13), 7885-7893. http://dx.doi.org/10.1103/PhysRevB.42.7885

\section{Copyrights}

Copyright for this article is retained by the author(s), with first publication rights granted to the journal.

This is an open-access article distributed under the terms and conditions of the Creative Commons Attribution license (http://creativecommons.org/licenses/by/3.0/). 